[Article]

\title{
$\mathrm{H}_{2}$ XP...SHY 复合物中磷键与硫键的理论研究
}

\author{
刘玉震黎安勇* \\ (西南大学化学化工学院, 重庆 400715)
}

\begin{abstract}
摘要: 用从头算量子化学方法 MP2 与 $\operatorname{CCSD}(T)$ 研究了 $\mathrm{H}_{2} X P$ 和 $S H Y(X, Y=H, F, C l, B r)$ 分子的 $P$ 与 $S$ 之间形 成的磷键 $X-P \cdots S$ 与硫键 $Y-S \cdots P$ 的本质与规律以及取代基 $X$ 与 $Y$ 对成键的影响. 计算结果表明, 硫键比磷 键强, 连接在 Lewis 酸上的取代基的电负性增大导致形成的磷键或硫键增强, 键能增大, 对单体的结构和性质 的影响也增大; 而连接在 Lewis 碱上的取代基效应则相反. 硫键键能为 $8.37-23.45 \mathrm{~kJ} \cdot \mathrm{mol}^{-1}$, 最强的硫键结构 是 $Y$ 电负性最大而 $X$ 电负性最小的 $\mathrm{HFS} \cdots \mathrm{PH}_{3}, \mathrm{CCSD}(\mathrm{T})$ 计算的键能是 $16.04 \mathrm{~kJ} \cdot \mathrm{mol}^{-1}$; 磷键键能为 $7.54-$ $14.65 \mathrm{~kJ} \cdot \mathrm{mol}^{-1}$, 最强的磷键结构是 $X$ 电负性最大而 $Y$ 电负性最小的 $\mathrm{H}_{2} \mathrm{FP} \cdots \mathrm{SH}_{2}, \operatorname{CCSD}(\mathrm{T})$ 计算的键能是 $12.52 \mathrm{~kJ} \cdot \mathrm{mol}^{-1}$. 对磷键与硫键能量贡献较大的是交换与静电作用. 分子间超共轭 $I p(S)-\sigma^{*}(P X)$ 与 I $(P)-\sigma^{*}(S Y)$ 对磷键与硫键的形成起着重要作用, 它导致单体的极化, 其中硫键的极化效应较大, 从而有一定的共价特征.
\end{abstract}

关键词: $\sigma$-hole; 磷键; 硫键; 取代基效应

中图分类号: 0641

\section{Theoretical Analysis of Pnicogen and Chalcogen Bonds in $\mathrm{H}_{2}$ XP...SHY Complexes}

\author{
LIU Yu-Zhen LI An-Yong* \\ (School of Chemistry and Chemical Engineering, Southwest University, Chongqing 400715, P. R. China)
}

\begin{abstract}
The MP2 and $\operatorname{CCSD}(\mathrm{T})$ ab initio quantum chemistry methods were applied to study the pnicogen bonds $\mathrm{X}-\mathrm{P} \cdots \mathrm{S}$ and chalcogen bonds $\mathrm{Y}-\mathrm{S} \cdots \mathrm{P}$ formed between $\mathrm{PH}_{2} \mathrm{X}$ and $\mathrm{SHY}(\mathrm{X}, \mathrm{Y}=\mathrm{H}, \mathrm{F}, \mathrm{Cl}, \mathrm{Br}$ ) and the effects of the substituents $X$ and $Y$ on the bonds. Calculated results show that the chalcogen bonds are stronger than the pnicogen bonds. Strongly electronegative substituents that are connected to the Lewis acid strengthened the bonds and significantly affected the structures and properties of the monomers. Conversely, the substituents connected to the Lewis bases produced opposite effects. The energies of chalcogen bonds were $8.37-23.45 \mathrm{~kJ} \cdot \mathrm{mol}^{-1}$; the strongest chalcogen bond was found in the structure HFS-PH${ }_{3}$ using the CCSD ( $\mathrm{T}$ ) method with a bonding energy of $16.04 \mathrm{~kJ} \cdot \mathrm{mol}^{-1}$. The energies of pnicogen bonds were in the range 7.54$14.65 \mathrm{~kJ} \cdot \mathrm{mol}^{-1}$; the strongest pnicogen bond was found in $\mathrm{H}_{2} \mathrm{FP}-\mathrm{SH}_{2}$ using $\operatorname{CCSD}(\mathrm{T})$ with a bonding energy $12.52 \mathrm{~kJ} \cdot \mathrm{mol}^{-1}$. The most important factors for bond strength for both types of bonds were the exchange and electrostatic energies. The hyperconjugations $\mathrm{Ip}(\mathrm{S})-\sigma^{*}(\mathrm{PX})$ and $\mathrm{lp}(\mathrm{P})-\sigma^{*}(\mathrm{SY})$ play important roles in the formation of the pnicogen and chalcogen bonds, which both lead to polarization of the monomers. Polarization caused by the chalcogen bond is larger than that by the pnicogen bond, resulting in the chalcogen bond having less of a covalent character.
\end{abstract}

Key Words: $\sigma$-hole; Pnicogen bond; Chalcogen bond; Substituent effect

\section{1 引言}

非共价相互作用广泛存在于各种化学和生物
体系中, 并起着非常重要的作用,,$^{1-3}$ 它可以改变分子 的几何结构、电子结构与性质, 以及影响凝聚体系

Received: December 1, 2014; Revised: January 21, 2015; Published on Web: January 21, 2015.

"Corresponding author. Email: aylifnsy@swu.edu.cn; Tel: +86-23-68252360

(c) Editorial office of Acta Physico-Chimica Sinica 
的物理化学性质. 氢键是最早发现并得到广泛研究 的一种非共价相互作用, ${ }^{4,5}$ 随后双氢键 ${ }^{6}$ 和卤键 ${ }^{7-9}$ 也 先后被发现和深入研究. 近年来人们又发现了两种 新的非共价相互作用一一磷键和硫键, 它们分别是 $\mathrm{P} / \mathrm{As}$ 或 $\mathrm{S} / \mathrm{Se}$ 等第 $\mathrm{V}$ 主族或第 VI 主族原子作为 Lewis 酸与其它的富电子原子或基团作为 Lewis 碱 形成的分子间弱相互作用. ${ }^{10-12}$ 特别是 $\mathrm{P} 、 \mathrm{~S}$ 作为生命 体的重要组成元素, 它们所形成的非共价键对生物 体中核酸、蛋白质等有机大分子的空间结构和生理 活性都产生很大影响.

所有的非共价相互作用都有一个共同特征: 它 们都可以看作 Lewis 酸碱的作用, 在 Lewis 酸中存在 正静电势区域, 称为 $\sigma$-hole, 而 Lewis 碱中有负静电 势区域, Lewis 酸碱正负静电势区域的静电吸引往 往是形成非共价相互作用的决定性因素. $\sigma$-hole 是 Politzer $^{13}$ 和 Murray ${ }^{14}$ 等在研究了许多 $\mathrm{R}-\mathrm{X}$ 卤键的 性质之后首次提出的: 由于卤族原子 $\mathrm{X}$ 的 $p_{z}$ 孤对轨 道电子参与形成 $\mathrm{R}-\mathrm{X}$ 键, 使得 $\mathrm{X}$ 原子 $p_{z}$ 轨道外层 电子缺失, 这样就会形成 $\sigma$-hole, 基团 $\mathrm{R}$ 的吸电子能 力越强, $\sigma$-hole 也越强. Scheiner ${ }^{15}$ 的工作使得磷键受 到重视, 他研究了不同取代基 X对 XP $\cdots \mathrm{NH}_{3}$ 复合物 分子间磷键的影响, 并提出强吸电子基 $\mathrm{X}$ 能大大 增强 $\mathrm{P} \cdots \mathrm{N}$ 磷键的强度, 同时他又详细对比和总结 了磷键、硫键、卤键和氢键的形成特点与联系. ${ }^{16}$ Del Bene $e^{17,18}$ 等研究了 $\mathrm{H}_{2} \mathrm{XP} \cdots \mathrm{PCX} 、 \mathrm{H}_{2} \mathrm{FP} \cdots \mathrm{NH}_{2} \mathrm{~F}$ 等 复合物中相关磷键和氢键的结构特点与性质. 由于 吸电子基团 $\mathrm{X}$ 的存在, 在 $\mathrm{P}$ 原子附近 $\mathrm{XP}$ 延长线方向 上存在一个 $\sigma$-hole, 它的存在就是形成磷键的关键. 在磷键之后硫键也受到大量关注, ${ }^{19}$ 在 SHY 分子中, 吸电子取代基 $\mathrm{Y}$ 的存在同样使得 $\mathrm{S}$ 原子周围、YS 延 长线方向存在一个 $\sigma$-hole, 它使得 SHY 可与 Lewis 碱形成硫键.

本文中我们研究 $\mathrm{H}_{2} \mathrm{XP}$ 和 $\mathrm{SHY}(\mathrm{X}, \mathrm{Y}=\mathrm{F}, \mathrm{Cl}, \mathrm{Br})$ 之间形成的磷键和硫键复合物的结构、性质与成键 机理. 这两种分子中既有 $\sigma$-hole 作为 Lewis 酸, 也有 孤对电子作为 Lewis 碱, 因此它们之间可以形成氢 键 $\mathrm{P} / \mathrm{X} \cdots \mathrm{HS} 、 \mathrm{~S} / \mathrm{Y} \cdots \mathrm{HP}$, 卤键 $\mathrm{X} \cdots \mathrm{S} / \mathrm{Y} 、 \mathrm{Y} \cdots \mathrm{P} / \mathrm{X}$, 磷键 $X-P \cdots S / Y$, 以及硫键 $Y-S \cdots P / X$ 等多种非共价作 用, 但本文仅研究在 $P$ 与 $S$ 之间形成的磷键 $X-P \cdots S$ 或硫键 $Y-S \cdots P$, 考察取代基对该磷键和硫键的影 响以及对复合物 $\mathrm{H}_{2} \mathrm{XP} \cdots \mathrm{SHY}$ 的结构和性质的影响.

\section{2 计算方法}

采用二阶微扰理论 MP2 方法与 aug-cc-pVDZ 和 aug-cc-pVTZ 基组对所有单体和复合物进行了构 型优化和频率计算, 并在 aug-cc-pVTZ 基组优化构 型基础上用 $\operatorname{CCSD}(\mathrm{T}) / \mathrm{aug}-\mathrm{cc}-\mathrm{pVTZ}$ 方法做了单点能 计算. 相互作用能的计算采用了超分子方法, 并用 Boys 和 Bernardi 提出的 Counterpoise 方法 ${ }^{20}$ 做了基 组重叠误差 (BSSE)矫正. 所有的上述计算都是在 Gaussian 09 软件包 ${ }^{21}$ 中完成的. 电子密度拓扑分析 使用了 AIMAll 软件, ${ }^{22}$ 自然键轨道 $(\mathrm{NBO})$ 分析结果 使用了 GenNBO 5.0 程序. ${ }^{23}$ 定域分子轨道能量分解 分析(LMOEDA) ${ }^{24}$ 采用了 GAMESS.64 程序. ${ }^{25}$

\section{3 结果分析与讨论}

\section{1 磷键与硫键的形成}

由于 $\mathrm{XP}$ 与 $\mathrm{YS}$ 延长线处 $\sigma$-hole 的存在, $\mathrm{H}_{2} \mathrm{XP}$ 和 SHY 可以形成两种 P $\cdots$ S 相互作用的复合物构型: 一种是磷键构型 $\mathrm{X}-\mathrm{P}^{\delta+} \cdots \mathrm{S}^{\delta-}, \mathrm{H}_{2} \mathrm{XP}$ 是 Lewis 酸, SHY 为 Lewis 碱, $S$ 提供孤对电子; 另一种是硫键构 型 $\mathrm{Y}-\mathrm{S}^{\delta+} \cdots \mathrm{P}^{\delta-}$, SHY 是 Lewis 酸, $\mathrm{PH}_{2} \mathrm{X}$ 为 Lewis 碱, $\mathrm{P}$ 提供孤对电子. 图 1中给出了这两类复合物的结构 图. 未取代的 $\mathrm{H}_{3} \mathrm{P}$ 和 $\mathrm{H}_{2} \mathrm{~S}$ 不能形成 $\mathrm{P} \cdots \mathrm{S}$ 复合物, 因为 除了 $\mathrm{H}$ 原子附近为正静电势外, $\mathrm{P}$ 与 $\mathrm{S}$ 原子附近都 为负的静电势. 本文研究了以下几类体系. 第一类, $\mathrm{H}_{2} \mathrm{X}-\mathrm{P} \cdots \mathrm{SH}_{2}(\mathrm{X}=\mathrm{F}, \mathrm{Cl}, \mathrm{Br}), \mathrm{P}$ 有 $\sigma$-hole 而 $\mathrm{S}$ 没有, 只 能形成磷键, 记为 $P 1$, 研究磷氢化物的取代基 $X$ 的 改变对磷键的影响. 第二类, $\mathrm{HY}-\mathrm{S} \cdots \mathrm{PH}_{3}(\mathrm{Y}=\mathrm{F}, \mathrm{Cl}$, $\mathrm{Br}), \mathrm{S}$ 有 $\sigma$-hole 而P 没有, 只能形成硫键, 记为 $\mathrm{S}$, 研 究硫氢化物的取代基 $Y$ 的改变对硫键的影响. 第三 类, $\mathrm{H}_{2} \mathrm{XP} \cdots \mathrm{SYH}(\mathrm{X}, \mathrm{Y}=\mathrm{F}, \mathrm{Cl}, \mathrm{Br}), \mathrm{P}$ 与 $\mathrm{S}$ 都有 $\sigma$-hole 与孤对电子, 可以形成 $\mathrm{P}$ 键与 $\mathrm{S}$ 键. 该类研究了三种 结构: 磷键结构 $\mathrm{H}_{2} \mathrm{XP} \cdots \mathrm{SFH}(\mathrm{X}=\mathrm{F}, \mathrm{Cl}, \mathrm{Br})$, 研究 X 取 代基的影响并与 (1) 类比较, 记为 $\mathrm{P} 2$; 硫键结构 $\mathrm{HYS} \cdots \mathrm{PFH}_{2}(\mathrm{Y}=\mathrm{F}, \mathrm{Cl}, \mathrm{Br})$, 研究 $\mathrm{Y}$ 取代基的影响并 与(2) 类比较, 记为 $\mathrm{S} 2$; 对称结构 $\mathrm{H}_{2} \mathrm{XP} \cdots \mathrm{SXH}(\mathrm{X}=\mathrm{F}$, $\mathrm{Cl}, \mathrm{Br})$, 既有磷键也有硫键, 比较这两种键, 分别记

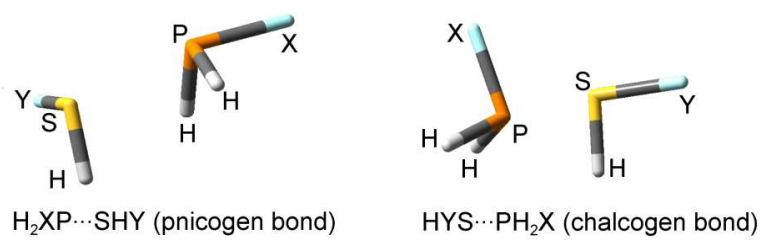

图 1 复合物的两种构型: 磷键与硫键

Fig.1 Two types of complexes: pnicogen bond and chalcogen bond 


\section{为 $\mathrm{P} 3$ 与 $\mathrm{S} 3$.}

图 2 中给出了 $\mathrm{MP} 2 /$ aug-cc-pVDZ 计算下的 $\mathrm{PH}_{2} \mathrm{Cl}$ 和 $\mathrm{SHCl}$ 单体分子在 0.0004 a.u. 等电子密度面的静 电势图, 图中颜色由红到蓝表示静电势值从负到正 逐渐增大. 分子静电势 (MEP) 图形表明 $\mathrm{PH}_{2} \mathrm{Cl}$ 和 $\mathrm{SHCl}$ 分子中 $\mathrm{ClP}$ 与 $\mathrm{ClS}$ 延长线上都明显具有一处正 静电势区域, 这就是 $\sigma$-hole. 用 Multiwfn 软件 ${ }^{26}$ 计算 的单体分子范德瓦尔斯面上 $\sigma$-hole 区域最大正静电 势值: 对 $\mathrm{PH}_{2} \mathrm{~F} 、 \mathrm{PH}_{2} \mathrm{Cl}$ 和 $\mathrm{PH}_{2} \mathrm{Br}$ 分别为 $180 、 159$ 和 $151 \mathrm{~kJ} \cdot \mathrm{mol}^{-1}$; 而对 $\mathrm{SHF} 、 \mathrm{SHCl}$ 和 $\mathrm{SHBr}$ 依次为 201 、 151 和 $130 \mathrm{~kJ} \cdot \mathrm{mol}^{-1}$. 因此, 对于 $\mathrm{PH}_{2} \mathrm{X}$ 和 $\mathrm{SHY}$ 单体, 随着取代卤素原子 $\mathrm{X} 、 \mathrm{Y}$ 从 $\mathrm{F} 、 \mathrm{Cl} 、 \mathrm{Br}$ 的递变, 取代基

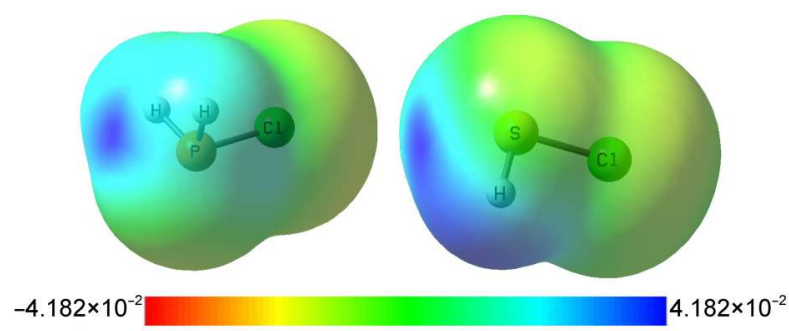

图 $2 \mathrm{PH}_{2} \mathrm{Cl}$ (左)和 $\mathrm{SHCl}$ (右)单体分子在 0.0004 a.u. 等 电子密度面上分子静电势分布

Fig.2 Molecular electrostatic potential (MEP) distribution of $\mathrm{PH}_{2} \mathrm{Cl}$ (left) and $\mathrm{SHCl}$ (right) on 0.0004 a.u. isosurface
电负性逐渐减小, 其 $\sigma$-hole 区域最大静电势值逐渐 减小, 亲电性依次降低. 取代基的吸电子能力越强, $\sigma$-hole 越强, 相应地, 其形成的磷键或硫键就越稳 定. 图 2 显示 $\mathrm{PH}_{2} \mathrm{Cl}$ 与 $\mathrm{SHCl}$ 的静电势分布呈现明显 不同, 对于相同的取代基 $\mathrm{X}=\mathrm{Y}, \mathrm{S}$ 的 $\sigma$-hole 强于 $\mathrm{P}$ 的 $\sigma$-hole, 由此可以预计硫键强于对应的磷键, 下文的 数据支持了这一预言.

\section{2 几何结构、频率与相互作用能}

表 1列出了用 MP2/aug-cc-pVDZ方法计算的各 类磷键与硫键结构的主要结构参数(单体间 $\mathrm{P} \cdots \mathrm{S}$ 距 离, 磷键或硫键作用角度 $\angle \mathrm{XPS}$ 或 $\angle \mathrm{YSP}) 、$ 键指数 $(P S 、 X P$ 与 $Y S$ 的 Wiberg 键级 $(\mathrm{WBI})$ ) 以及复合物形 成时 $P X$ 或 $S Y$ 键长与振动频率的变化. 表 1 也列出 了用 MP2/aug-cc-pVDZ、MP2/aug-cc-pVTZ和 CCSD (T)/aug -cc-pVTZ//MP2/aug-cc-pVTZ三种方法计算 的经过 BSSE 矫正的相互作用能, 分别记为 $E_{1} 、 E_{2}$ 和 $E_{3}$. 另外也用完全基组方法 CBS-QB3 计算了单体与 复合物的能量, 用此方法计算的相互作用能记为 $E_{4}$, 也列于表 1 中. 关于单体与复合物更加详细的结 构数据列在 Supporting Information 表 S1 中. 不同方 法计算的相互作用能有一定的差别, 其中完全基组 方法 CBS-QB3 计算的作用能最小, MP2/aug-cc$\mathrm{pVTZ}$ 计算的作用能最大, 其他两种方法的作用能 比较接近. 由于没有获得相应的实验数据, 很难判

表 1 用 MP2/aug-cc-pVDZ 方法计算的各种 $\mathrm{H}_{2} \mathrm{XP} \cdots \mathrm{SHY}$ 复合物的主要结构参数和性质与四种方法计算的相互作用能 Table 1 Main structural parameters of $\mathrm{H}_{2} X P \cdots$ SHY complexes at MP2/aug-cc-pVDZ level and interaction energies calculated using four methods

\begin{tabular}{|c|c|c|c|c|c|c|c|c|c|c|}
\hline Pnicogen bond & $L(\mathrm{P} \cdots \mathrm{S}) / \mathrm{nm}$ & $\angle \mathrm{XPS} /\left(^{\circ}\right)$ & WBI(PS) & $\mathrm{WBI}(\mathrm{PX})$ & $\Delta L(\mathrm{PX}) / \mathrm{nm}$ & $\Delta v(\mathrm{PX}) / \mathrm{cm}^{-1}$ & $E_{1} /\left(\mathrm{kJ} \cdot \mathrm{mol}^{-1}\right)$ & $E_{2} /\left(\mathrm{kJ} \cdot \mathrm{mol}^{-1}\right.$ & $E_{3} /\left(\mathrm{kJ} \cdot \mathrm{mol}^{-1}\right.$ & $\left.{ }^{1}\right) E_{4} /\left(\mathrm{kJ} \cdot \mathrm{mol}^{-1}\right)$ \\
\hline $\mathrm{H}_{2} \mathrm{FP}_{-} \mathrm{SH}_{2}(\mathrm{P} 1)$ & 0.3254 & 166.7 & 0.057 & 0.570 & 0.0010 & -25.7 & -12.69 & -14.44 & -12.52 & -10.12 \\
\hline $\mathrm{H}_{2} \mathrm{ClP}-\mathrm{SH}_{2}(\mathrm{P} 1)$ & 0.3324 & 166.5 & 0.047 & 0.853 & 0.0015 & -21.0 & -11.43 & -12.85 & -10.84 & -8.32 \\
\hline $\mathrm{H}_{2} \mathrm{BrP}_{-} \mathrm{SH}_{2}(\mathrm{P} 1)$ & 0.3338 & 166.3 & 0.045 & 0.905 & 0.0016 & -16.7 & -10.89 & -12.35 & -10.09 & -9.61 \\
\hline $\mathrm{H}_{2} \mathrm{FP}-\mathrm{SHF}(\mathrm{P} 2, \mathrm{P} 3)$ & 0.3228 & 168.1 & 0.055 & 0.575 & 0.0006 & -18.5 & -9.21 & -12.23 & -10.30 & -7.05 \\
\hline $\mathrm{H}_{2} \mathrm{ClP}-\mathrm{SHF}(\mathrm{P} 2)$ & 0.3333 & 167.3 & 0.038 & 0.864 & 0.0009 & -13.3 & -8.16 & -10.51 & -8.67 & -5.20 \\
\hline $\mathrm{H}_{2} \mathrm{BrP}-\mathrm{SHF}(\mathrm{P} 2)$ & 0.3357 & 166.4 & 0.035 & 0.917 & 0.0010 & -9.7 & -7.87 & -9.96 & -8.08 & -6.16 \\
\hline $\mathrm{H}_{2} \mathrm{ClP}-\mathrm{SHCl}(\mathrm{P} 3)$ & 0.3285 & 167.3 & 0.041 & 0.862 & 0.0011 & -16.0 & -11.39 & -13.98 & -10.89 & -6.40 \\
\hline$\underline{\mathrm{H}_{2} \mathrm{BrP}-\mathrm{SHBr}(\mathrm{P} 3)}$ & 0.3278 & 167.0 & 0.041 & 0.911 & 0.0013 & -13.3 & -11.89 & -14.65 & - & - \\
\hline Chalcogen bond & $L(\mathrm{P} \cdots \mathrm{S}) / \mathrm{nm}$ & $\angle \mathrm{YSP} /\left(^{\circ}\right)$ & WBI(PS) & WBI(SY) & $\Delta L(\mathrm{SY}) / \mathrm{nm}$ & $\Delta v(\mathrm{SY}) / \mathrm{cm}^{-1}$ & $E_{1} /\left(\mathrm{kJ} \cdot \mathrm{mol}^{-1}\right)$ & \multicolumn{3}{|c|}{$E_{2} /\left(\mathrm{kJ} \cdot \mathrm{mol}^{-1}\right) E_{3} /\left(\mathrm{kJ} \cdot \mathrm{mol}^{-1}\right) E_{4} /\left(\mathrm{kJ} \cdot \mathrm{mol}^{-1}\right)$} \\
\hline HFS- $\mathrm{PH}_{3}(\mathrm{~S} 1)$ & 0.2889 & 173.8 & 0.160 & 0.649 & 0.0027 & -80.2 & -18.25 & -23.40 & -16.04 & -15.80 \\
\hline $\mathrm{HClS}-\mathrm{PH}_{3}(\mathrm{~S} 1)$ & 0.3166 & 172.6 & 0.063 & 0.932 & 0.0020 & -37.0 & -12.73 & -14.99 & -11.26 & -7.65 \\
\hline $\mathrm{HBrS}_{-} \mathrm{PH}_{3}(\mathrm{~S} 1)$ & 0.3220 & 172.1 & 0.052 & 0.968 & 0.0019 & -27.9 & -11.60 & -13.65 & -9.96 & -7.60 \\
\hline HFS-PH ${ }_{2} \mathrm{~F}(\mathrm{~S} 2, \mathrm{~S} 3)$ & 0.2852 & 175.7 & 0.169 & 0.653 & 0.0024 & -76.5 & -13.65 & -22.99 & -10.51 & -17.95 \\
\hline $\mathrm{HClS}-\mathrm{PH}_{2} \mathrm{~F}(\mathrm{~S} 2)$ & 0.3186 & 175.7 & 0.055 & 0.942 & 0.0014 & -27.7 & -9.00 & -12.81 & -9.00 & -6.56 \\
\hline $\mathrm{HBrS}-\mathrm{PH}_{2} \mathrm{~F}(\mathrm{~S} 2)$ & 0.3235 & 175.8 & 0.044 & 0.977 & 0.0014 & -20.6 & -8.16 & -11.56 & -8.16 & -6.81 \\
\hline $\mathrm{HClS}-\mathrm{PH}_{2} \mathrm{Cl}(\mathrm{S} 3)$ & 0.3153 & 176.3 & 0.052 & 0.944 & 0.0015 & -31.0 & -9.92 & -13.98 & -8.79 & -8.20 \\
\hline $\mathrm{HBrS}-\mathrm{PH}_{2} \mathrm{Br}(\mathrm{S} 3)$ & 0.3181 & 176.7 & 0.044 & 0.978 & 0.0015 & -24.6 & -9.55 & -13.40 & - & -8.63 \\
\hline
\end{tabular}


断用哪种方法计算能量更好.

在磷键体系中磷键的形成导致 XP 键伸长以及 振动频率红移, 在硫键体系中硫键的形成导致 YS 键伸长及其振动频率红移, 这分别是由于分子间超 共轭 $n(\mathrm{~S}) \rightarrow \sigma^{*}(\mathrm{PX})$ 与 $n(\mathrm{P}) \rightarrow \sigma^{*}(\mathrm{SY})$ 产生的电子密度 从一个单体的孤对轨道转移到另一个单体的相关 反键 $\sigma^{*}$ 轨道导致 $\mathrm{PX}$ 或 SY 键削弱造成的. 硫键体系 的键长增大与频率红移大于磷键体系, 硫键体系中 $\mathrm{P} \cdots \mathrm{S}$ 距离比磷键体系的总体来说要小, 硫键的键能 总体上比磷键大 (对称体系 $\mathrm{P} 3$ 与 $\mathrm{S} 3$ 例外), 说明硫键 总体上比磷键强. 硫键作用角度 $\angle \mathrm{YSP}$ 大于磷键的 $\angle X P S$, 硫键更加接近于直线型, 这个差别可能与 $\mathrm{P}$ 和 $\mathrm{S}$ 的价态与配位数不同有关.

对 $\mathrm{P} 1 、 \mathrm{P} 2 、 \mathrm{~S} 1$ 与 $\mathrm{S} 2$ 型结构, 随着取代基 $\mathrm{X}$ 或 $\mathrm{Y}$ 按照 $\mathrm{F} 、 \mathrm{Cl}$ 与 $\mathrm{Br}$ 的顺序变化, $\mathrm{P} \cdots \mathrm{S}$ 距离依次递增, 键 指数 WBI(PS) 依次递减, WBI(PX) 与 WBI(SY) 依次 递增, $\mathrm{SY}$ 键的伸长与振动频率红移依次递减, 而 PX 键的伸长依次递增但振动频率红移依次递减, 相互 作用能依次减小. 这些(特别是相互作用能、P $\cdots \mathrm{S}$ 距 离以及 WBI 等数据) 说明形成的磷键或硫键按此顺 序越来越弱, 磷键或硫键的形成对 PX 或 SY 键的削 弱越来越弱以及对单体结构和性质的影响也越来 越弱, ${ }^{27}$ 这是 $\sigma$-hole 的强度按此顺序递减的结果. P2/ $\mathrm{S} 2$ 系列结构形成的磷键/硫键比 P $1 / \mathrm{S} 1$ 系列对应结 构的磷键/硫键弱, 这是 Lewis 碱上电负性大的取代
基 $\mathrm{F}$ 吸电子能力强导致 Lewis 碱的亲核性减弱的结 果.

对 P3 和 S3 型对称取代结构 $\mathrm{H}_{2} \mathrm{XP} \cdots \mathrm{SXH}$, 大电 负性的 $\mathrm{X}$ 原子大的吸电子能力使得 $\mathrm{P}$ (或 $\mathrm{S}$ )有强的 $\sigma$ hole, 但也使得 $\mathrm{S}$ (或 $\mathrm{P}$ ) 的亲核能力减弱, 这两个作用 彼此抵消, 最终磷键与硫键的强弱决定于这两个作 用的竞争. 从表 1 计算结果看, 对硫键 $\mathrm{S} 3$ 结构系列, 第一个作用整体上占优势, 随着取代基 $\mathrm{X}$ 按照 $\mathrm{F} 、 \mathrm{Cl}$ 与 $\mathrm{Br}$ 的顺序变化, $\mathrm{P} \cdots \mathrm{S}$ 距离依次递增, WBI(PS)依 次递减, WBI(SY) 依次递增, $\mathrm{SY}$ 键的伸长与振动频 率红移依次递减, 硫键键能依次递减. 而对磷键 P3 结构系列, 这两个作用的表现比较复杂, 任一个都 没有明显优势, 随着取代基 $\mathrm{X}$ 按照 $\mathrm{F} 、 \mathrm{Cl}$ 与 $\mathrm{Br}$ 的顺 序变化, WBI(PX)依次递增, PX 键伸长依次递增而 振动频率红移依次下降, 相互作用能依次递增.

此外, 在所有各系列的 $\mathrm{F} 、 \mathrm{Cl}$ 与 $\mathrm{Br}$ 取代的三个 结构的各种性质中, $\mathrm{Cl}$ 与 $\mathrm{Br}$ 取代结构的数值比较接 近, 而 $\mathrm{F}$ 取代结构的相应数值与之差距较大, 这是 $\mathrm{Cl}$ 和 $\mathrm{Br}$ 电负性接近而 $\mathrm{F}$ 比它们的电负性大得多的结 果.

\section{3 磷键与硫键的本质特征}

在 MP2/aug-cc-pVDZ 水平下对所有单体与复 合物进行了电子密度拓扑分析与自然间轨道分析, 电子密度临界点的性质列于表 2 中. 对于所有的磷 键和硫键系列, 单体间 $\mathrm{S} \cdots \mathrm{P}$ 临界点电子密度 $\rho<0.1$

表 2 用 MP2/aug-cc-pVTZ 计算的复合物中 $P \cdots S$ 与 $P X$ 和 $S Y$ 键临界点的性质

Table 2 Characteristics of $P \cdots S$, PX, and SY bond critical points calculated at MP2/aug-cc-pVDZ level

\begin{tabular}{|c|c|c|c|c|c|c|}
\hline Pnicogen bond & $\rho(\mathrm{P} \cdots \mathrm{S})$ & $\nabla^{2} \rho(\mathrm{P} \cdots \mathrm{S})$ & $H(\mathrm{P} \cdots \mathrm{S})$ & $\triangle \rho(\mathrm{PX})$ & $\nabla^{2} \rho(\mathrm{PX})$ & $H(\mathrm{PX})$ \\
\hline $\mathrm{H}_{2} \mathrm{FP}-\mathrm{SH}_{2}(\mathrm{P} 1)$ & 0.0141 & 0.0360 & 0.0002 & -0.0035 & 0.8302 & -0.0846 \\
\hline $\mathrm{H}_{2} \mathrm{ClP}-\mathrm{SH}_{2}(\mathrm{P} 1)$ & 0.0124 & 0.0323 & 0.0005 & -0.0033 & -0.0382 & -0.1016 \\
\hline $\mathrm{H}_{2} \mathrm{BrP}_{-} \mathrm{SH}_{2}(\mathrm{P} 1)$ & 0.0126 & 0.0320 & 0.0005 & -0.0033 & -0.1779 & -0.0825 \\
\hline $\mathrm{H}_{2} \mathrm{FP}-\mathrm{SHF}(\mathrm{P} 2, \mathrm{P} 3)$ & 0.0173 & 0.0414 & -0.0004 & -0.0026 & 0.8383 & -0.0854 \\
\hline $\mathrm{H}_{2} \mathrm{ClP}-\mathrm{SHF}(\mathrm{P} 2)$ & 0.0134 & 0.0350 & 0.0004 & -0.0023 & -0.0354 & -0.1029 \\
\hline $\mathrm{H}_{2} \mathrm{BrP}-\mathrm{SHF}(\mathrm{P} 2)$ & 0.0135 & 0.0346 & 0.0004 & -0.0023 & -0.1824 & -0.0842 \\
\hline $\mathrm{H}_{2} \mathrm{ClP}-\mathrm{SHCl}(\mathrm{P} 3)$ & 0.0135 & 0.0349 & 0.0003 & -0.0025 & -0.0352 & -0.1026 \\
\hline $\mathrm{H}_{2} \mathrm{BrP}-\mathrm{SHBr}(\mathrm{P} 3)$ & 0.0141 & 0.0354 & 0.0003 & -0.0027 & -0.1809 & -0.0836 \\
\hline Chalcogen bond & $\rho(\mathrm{P} \cdots \mathrm{S})$ & $\nabla^{2} \rho(\mathrm{P} \cdots \mathrm{S})$ & $H(\mathrm{P} \cdots \mathrm{S})$ & $\triangle \rho(\mathrm{SY})$ & $\nabla^{2} \rho(\mathrm{SY})$ & $H(\mathrm{SY})$ \\
\hline HFS-PH ${ }_{3}(\mathrm{~S} 1)$ & 0.0370 & 0.0640 & -0.0056 & -0.0131 & 0.2662 & -0.1636 \\
\hline $\mathrm{HClS}_{-} \mathrm{PH}_{3}(\mathrm{~S} 1)$ & 0.0193 & 0.0478 & -0.0013 & -0.0068 & -0.1701 & -0.0961 \\
\hline $\mathrm{HBrS}_{-} \mathrm{PH}_{3}(\mathrm{~S} 1)$ & 0.0180 & 0.0450 & 0.0010 & -0.0045 & -0.1074 & -0.0669 \\
\hline HFS-PH ${ }_{2} \mathrm{~F}(\mathrm{~S} 2, \mathrm{~S} 3)$ & 0.0653 & 0.0530 & -0.0200 & -0.0216 & 0.1358 & -0.1533 \\
\hline $\mathrm{HClS} \mathrm{PH}_{2} \mathrm{~F}(\mathrm{~S} 2)$ & 0.0215 & 0.0508 & -0.0006 & -0.0064 & -0.1716 & -0.0964 \\
\hline $\mathrm{HBrS}-\mathrm{PH}_{2} \mathrm{~F}(\mathrm{~S} 2)$ & 0.0197 & 0.0475 & -0.0002 & -0.0042 & -0.1084 & -0.0672 \\
\hline $\mathrm{HClS}-\mathrm{PH}_{2} \mathrm{Cl}(\mathrm{S} 3)$ & 0.0211 & 0.0510 & -0.0005 & -0.0058 & -0.1735 & -0.0970 \\
\hline $\mathrm{HBrS}-\mathrm{PH}_{2} \mathrm{Br}(\mathrm{S} 3)$ & 0.0197 & 0.0486 & -0.0002 & -0.0039 & -0.1093 & -0.0675 \\
\hline
\end{tabular}

$\rho$ : electron density, $\nabla^{2} \rho$ : Laplacian of electron density, $H$ : electronic energy density. All these quantities are in units of atomic units (a.u.). 
表 3 在 MP2/aug-cc-pVDZ 水平下计算的 LMOEDA 能量分解分析结果

Table 3 Energy decomposition analysis by LMOEDA calculated at MP2/aug-cc-pVDZ level

\begin{tabular}{|c|c|c|c|c|c|c|}
\hline System & ES & EX & REP & POL & MP2 DISP & MP2 \\
\hline $\mathrm{H}_{2} \mathrm{FP}-\mathrm{SH}_{2}(\mathrm{P} 1)$ & -27.97 & -55.01 & 91.44 & -12.10 & -14.36 & -18.00 \\
\hline $\mathrm{H}_{2} \mathrm{ClP}_{-} \mathrm{SH}_{2}(\mathrm{P} 1)$ & -24.20 & -49.82 & 81.94 & -10.47 & -14.11 & -16.66 \\
\hline $\mathrm{H}_{2} \mathrm{BrP}_{-} \mathrm{SH}_{2}(\mathrm{P} 1)$ & -23.57 & -49.66 & 81.48 & -10.26 & -13.94 & -15.99 \\
\hline $\mathrm{H}_{2} \mathrm{FP}-\mathrm{SHF}(\mathrm{P} 2, \mathrm{P} 3)$ & -21.10 & -48.78 & 81.68 & -11.14 & -15.70 & -15.03 \\
\hline $\mathrm{H}_{2} \mathrm{ClP}-\mathrm{SHF}(\mathrm{P} 2)$ & -16.08 & -39.15 & 64.64 & -8.37 & -14.74 & -13.65 \\
\hline $\mathrm{H}_{2} \mathrm{BrP}-\mathrm{SHF}(\mathrm{P} 2)$ & -15.28 & -37.93 & 62.51 & -7.91 & -14.53 & -13.15 \\
\hline $\mathrm{H}_{2} \mathrm{ClP}-\mathrm{SHCl}(\mathrm{P} 3)$ & -21.77 & -52.34 & 85.87 & -10.63 & -19.93 & -18.80 \\
\hline $\mathrm{H}_{2} \mathrm{BrP}-\mathrm{SHBr}(\mathrm{P} 3)$ & -23.61 & -58.36 & 95.67 & -11.47 & -22.19 & -20.05 \\
\hline HFS-PH ${ }_{3}(\mathrm{~S} 1)$ & -71.68 & -137.70 & 247.23 & -42.33 & -23.15 & -27.59 \\
\hline $\mathrm{HClS}-\mathrm{PH}_{3}(\mathrm{~S} 1)$ & -35.25 & -72.47 & 124.31 & -17.33 & -17.50 & -18.25 \\
\hline $\mathrm{HBrS}-\mathrm{PH}_{3}(\mathrm{~S} 1)$ & -30.52 & -64.81 & 110.45 & -14.82 & -16.66 & -16.33 \\
\hline HFS-PH ${ }_{2} \mathrm{~F}(\mathrm{~S} 2, \mathrm{~S} 3)$ & -68.33 & -140.51 & 254.60 & -44.55 & -25.08 & -23.86 \\
\hline HClS-PH ${ }_{2} \mathrm{~F}(\mathrm{~S} 2)$ & -26.08 & -60.21 & 103.20 & -14.57 & -16.91 & -14.61 \\
\hline $\mathrm{HBrS}-\mathrm{PH}_{2} \mathrm{~F}(\mathrm{~S} 2)$ & -21.86 & -52.92 & 90.18 & -12.27 & -16.24 & -13.10 \\
\hline $\mathrm{HClS}-\mathrm{PH}_{2} \mathrm{Cl}(\mathrm{S} 3)$ & -26.17 & -64.35 & 110.11 & -15.41 & -20.85 & -16.62 \\
\hline $\mathrm{HBrS}-\mathrm{PH}_{2} \mathrm{Br}(\mathrm{S} 3)$ & -23.15 & -61.76 & 104.96 & -13.82 & -22.44 & -16.16 \\
\hline
\end{tabular}

LMOEDA: localized molecular orbital energy decomposition analysis; ES: electrostatic energy, EX: exchange energy;

REP: repulsion energy, POL: polarization energy, DISP: dispersion energy. All these quantities are in $\mathrm{kJ} \cdot \mathrm{mol}^{-1}$.

a.u. 而拉普拉斯 $\nabla^{2} \rho$ 在 $0-0.1$ a.u.之间, 因此磷键和硫 键都是弱的相互作用, 但硫键的电子密度大于磷键 的电子密度, 说明硫键更强, 与前面的分析一致. 所 有磷键的 $\mathrm{S} \cdots \mathrm{P}$ 临界点的电子能量密度 $H$ 都是一个 很小的正值 (一个例外), 而所有硫键的临界点的电 子能量密度 $H$ 则略大些, 是一个小的负值 $($ 一个例 外). 因此大致可以说硫键有很小的共价特征. 取代 基在硫键系列 $\mathrm{S} 1 、 \mathrm{~S} 2$ 与 $\mathrm{S} 3$ 的效应有明显的规律, 即 从 $\mathrm{F}$ 到 $\mathrm{Cl}$ 和 $\mathrm{Br}, \mathrm{S} \cdots \mathrm{P}$ 临界点电子密度与拉普拉斯都 减小, 表明硫键减弱, 与前面的结果一致. 而对于磷 键系列, 则取代基的效应没有明显的规律.

磷键或硫键的形成对单体中化学键 PX 与 SY 的影响有一定的规律性. 对磷键系列, 分子间磷键 的形成会导致 PX 键临界点电子密度降低, 且该降 低的趋势会随着电子供体 $\mathrm{SH}_{2}$ 分子内的氢原子被卤 族原子 $\mathrm{X}(\mathrm{F} 、 \mathrm{Cl} 、 \mathrm{Br})$ 的取代而有所削弱. 分子间硫键 的形成也具有同样的影响规律, 且 SY 键临界点 电子密度下降的幅度更大(只有 HFS $\cdots \mathrm{PH}_{2} \mathrm{~F}$ 构型 中 $\rho(\mathrm{SY})$ 比 $\mathrm{HFS} \cdots \mathrm{PH}_{3}$ 中稍低).

自然键轨道分析表明, 在磷键复合物中, 有电 子密度从 $\mathrm{S}$ 孤对轨道 $\mathrm{l}\left(\mathrm{p}(\mathrm{S})\right.$ 向 $\sigma^{*}(\mathrm{PX})$ 反键轨道转移, 导致 $\operatorname{lp}(\mathrm{S})$ 占据减小而 $\sigma^{*}(\mathrm{PX})$ 占据增大, 从而 $\mathrm{PX}$ 键 伸长并且振动频率红移, 分子间超共轭的二阶稳定 化能 $E^{(2)}\left\{\mathrm{lp}(\mathrm{S})-\sigma^{*}(\mathrm{PX})\right\}$ 约为 $31.4-44.0 \mathrm{~kJ} \cdot \mathrm{mol}^{-1}$, 分 子间电荷转移大致为 0.03 a.u. 左右. 在硫键复合物
中, 存在电子密度从 $\mathrm{P}$ 孤对轨道 $\operatorname{lp}(\mathrm{P})$ 向 $\sigma^{*}(\mathrm{SY})$ 反键 轨道转移, 导致 $\operatorname{lp}(\mathrm{P})$ 占据减小而 $\sigma^{*}(\mathrm{SY})$ 占据增大, 因而 $\mathrm{SY}$ 键长伸长并且振动频率红移, 分子间超共 轭的二阶稳定化能 $E^{(2)}\left\{\mathrm{l}(\mathrm{P})-\sigma^{*}(\mathrm{SY})\right\}$ 较大, 约为 41.9-132.3 kJ $\cdot \mathrm{mol}^{-1}$, 分子间电荷转移也较大 $(0.04-$ 0.22 a.u.), 这些数据也表明硫键比磷键强.

为进一步考察磷键与硫键的本质, 我们在 MP2/ aug-cc-pVDZ 水平下用定域分子轨道能量分解分析 方法计算了所有磷键与硫键结构的相互作用能的 各个成分(没有进行 BSSE 校正), 结果列于表 3 中. 排斥能(REP) 总是绝对值最大的项. 在静电能(ES)、 交换能(EX)、极化能(POL)与色散能(DISP)四个吸引 项中交换项 EX贡献最大, 达到总吸引能的 $50 \%$, 其 次是静电项, 贡献为 $20 \%-26 \%$, 第三是 DISP 色散项 贡献为 $13 \%-19 \%$, 最小是 POL 极化项贡献为 $10 \%-$ $12 \%$. 例外的是两个含 $\mathrm{F}$ 的硫键结构 $\mathrm{HFS} \cdots \mathrm{PH}_{3}$ 与 $\mathrm{HFS} \cdots \mathrm{PH}_{2} \mathrm{~F}$, 其中极化能占总吸引能的 $15 \%-16 \%$, 而 DISP 色散能的贡献仅为 $8 \%-9 \%$. 这些结果与磷 键和硫键的一般特征吻合, LMOEDA 分析给出的磷 键和硫键的一般规律是: 排斥项绝对值最大, 交换 项对总吸引能的贡献最大, 一般达到 $50 \%$, 其次是 静电项, 贡献一般大于 $20 \%$.

\section{4 结 论}

从理论上研究了三个磷键与三个硫键系列 
$\mathrm{H}_{2} \mathrm{XP} \cdots \mathrm{SHY}(\mathrm{X}, \mathrm{Y}=\mathrm{H}, \mathrm{F}, \mathrm{Cl}, \mathrm{Br})$ 的结构、键能、频率 与成键特征, 发现 $\mathrm{P}$ 或 $\mathrm{S}$ 上的取代基对磷键和硫键 的影响具有良好的规律性. 随着取代基 $X($ 或 $Y$ )的电 负性增大, $\mathrm{P}$ (或 $\mathrm{S}$ )的 $\sigma$-hole 的亲电性增强, 形成的磷

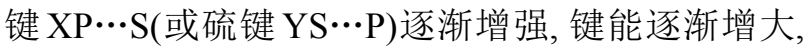
单体间距离 $\mathrm{P} \cdots \mathrm{S}$ 减小, $\mathrm{PX}$ (或 SY) 键的伸缩振动频 率红移逐渐增大, 磷键 (或硫键)对单体结构与性质 的影响越来越大. 总体来说, 硫键比磷键强, 硫键作 用更接近于线性并且有小的共价特征. 对磷键与硫 键作用能贡献较大的是交换与静电作用. 磷键与硫 键都是给体一受体相互作用, 这种作用导致两个单 体有一定程度的极化.

Supporting Information: Some structural parameters of all monomers and complexes are listed in Table S1. This information is available free of charge via the internet at http://www. whxb.pku.edu.cn.

\section{References}

(1) Buckingham, A. D.; Fowler, P. W.; Hutson, J. M. Chem. Rev. 1988, 88, 963. doi: 10.1021/cr00088a008

(2) Chalasinski, G.; Szczesniak, M. M. Chem. Rev. 2000, 100, 4227. doi: $10.1021 / \mathrm{cr} 990048 \mathrm{z}$

(3) Wormer, P. E. S.; van der Avoird, A. Chem. Rev. 2000, 100, 4109. doi: $10.1021 / \mathrm{cr} 990046 \mathrm{e}$

(4) Jeffrey, G. A. Crystal. Rev. 2003, 9, 135. doi: 10.1080/ 08893110310001621754

(5) Gilli, G.; Gilli, P. The Nature of the Hydrogen Bond; Oxford University Press: Oxford, UK, 2009; pp 2-51.

(6) Bakhmutov, V. I. Dihydrogen Bond: Principles, Experiments, and Applications; Wiley-Interscience: New York, 2008.

(7) Politzer, P.; Murray, J. S.; Clark, T. Phys. Chem. Chem. Phys. 2010, 12, 7748. doi: 10.1039/c004189k

(8) Brammer, L.; Espallargas, G. M.; Libri, S. CrystEngComm 2008, 10, 1712. doi: 10.1039/b812927d
(9) Politzer, P.; Murray, J. S.; Clark, T. Phys. Chem. Chem. Phys. 2013, 15, 11178. doi: 10.1039/c3cp00054k

(10) Scheiner, S. CrystEngComm 2013, 15, 3119. doi: 10.1039/ c2ce26393a

(11) Guan, L. Y.; Mo, Y. R. J. Phys. Chem. A 2014, 118, 8911. doi: 10.1021/jp500775m

(12) Murray, J. S.; Lane, P.; Clark, T.; Politzer, P. J. Mol. Model. 2007, 13, 1033. doi: 10.1007/s00894-007-0225-4

(13) Clark, T.; Hennemann, M.; Murray, J. S.; Politzer, P. J. Mol. Model. 2007, 13, 291. doi: 10.1007/s00894-006-0130-2

(14) Murray, J. S.; Lane, P.; Politzer, P. J. Mol. Model. 2009, 15, 723. doi: 10.1007/s00894-008-0386-9

(15) Scheiner, S. J. Phys. Chem. A 2011, 115, 11202. doi: 10.1021/ jp203964b

(16) Scheiner, S. International Journal of Quantum Chemistry 2013, 113, 1609. doi: 10.1002/qua.v113.11

(17) Alkorta, I.; Sánchez-Sanz, G.; Elguero, J.; Del Bene, J. E. J. Phys. Chem. A 2013, 117, 183. doi: 10.1021/jp3100816

(18) Del Bene, J. E.; Alkorta, I.; Elguero, J. Theor. Chem. Acc. 2014, 133, 1464. doi: 10.1007/s00214-014-1464-y

(19) Adhikari, U.; Scheiner, S. J. Phys. Chem. A 2014, 118, 3183.

(20) Boys, S. F.; Bernardi, F. Mol. Phys. 1970, 19, 553.

(21) Frisch, M. J.; Trucks, G. W.; Schlegel, H. B.; et al. Gaussian 09, Revision A.02; Gaussian: Wallingford, CT, 2009.

(22) Keith, T. A. AIMAll, Version 13.10.19; TK Gristmill Software, Overland Park KS, USA, 2013. (aim.tkgristmill.com).

(23) Glendening, E. D.; Badenhoop, J. K.; Reed, A. E.; Carpenter, J. E.; Bohmann, J. A.; Weinhold, F. GENNBO5.0W; Theoretical Chemistry Institute: University of Wisconsin, Madison, WI, 2001.

(24) Su, P.; Li, H. J. Chem. Phys. 2009, 131, 014102. doi: 10.1063/ 1.3159673

(25) Schmidt, M. W.; Baldridge, K. K.; Boatz, J. A. J. Comput. Chem. 1993, 14, 1347.

(26) Lu, T.; Chen, F. J. J. Comput. Chem. 2012, 33, 580. doi: $10.1002 /$ jcc.v33.5

(27) Zhao, G.; Han, K. Accounts Chem. Res. 2012, 45, 404. doi: $10.1021 / \mathrm{ar} 200135 \mathrm{~h}$ 
Supplementary Information for Acta Phys. -Chim. Sin. 2015, 31 (3), 435-440 doi: 10.3866/PKU.WHXB201501211

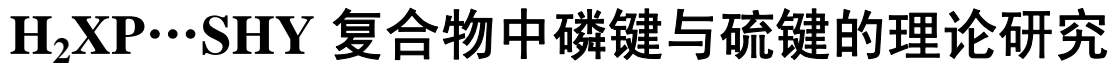

\author{
刘玉震＼cjkstart黎安勇* \\ (西南大学化学化工学院, 重庆 400715)
}

\section{Theoretical Analysis of Pnicogen and Chalcogen Bonds in $\mathrm{H}_{2}$ XP...SHY Complexes}

\author{
LIU Yu-Zhen LI An-Yong*
}

(School of Chemistry and Chemical Engineering, Southwest University, Chongqing 400715, P. R. China)

*Corresponding author. Email: aylifnsy@swu.edu.cn; Tel: +86-23-68252360 
表 S1 各单体和复合物的相关结构参数

Table S1 Some structural parameters of all monomers and complexes

\begin{tabular}{|c|c|c|c|c|c|}
\hline Pnicogen bond & $L(\mathrm{PH}) / \mathrm{nm}$ & $L(\mathrm{PX}) / \mathrm{nm}$ & $v(\mathrm{PX}) / \mathrm{cm}^{-1}$ & $L(\mathrm{P} \cdots \mathrm{S}) / \mathrm{nm}$ & $\angle \mathrm{XPS} /\left(^{\circ}\right)$ \\
\hline $\mathrm{H}_{2} \mathrm{FP}$ & $0.1416(0.1430)$ & $0.1622(0.1673)$ & 809(778.9) & & \\
\hline $\mathrm{H}_{2} \mathrm{ClP}$ & $0.1413(0.1427)$ & $0.2076(0.2114)$ & 523.2(501.2) & & \\
\hline $\mathrm{H}_{2} \mathrm{BrP}$ & $0.1413(0.1427)$ & $0.2234(0.2272)$ & 415.5(396.9) & & \\
\hline $\mathrm{H}_{2} \mathrm{FP}_{-} \mathrm{SH}_{2}(\mathrm{P} 1)$ & $0.1415(0.1429)$ & $0.1631(0.1683)$ & 780.2(753.2) & 0.3192 & 166.4 \\
\hline $\mathrm{H}_{2} \mathrm{ClP}_{-} \mathrm{SH}_{2}(\mathrm{P} 1)$ & $0.1412(0.1425)$ & $0.2090(0.2129)$ & $500.5(480.2)$ & 0.3283 & 165.9 \\
\hline $\mathrm{H}_{2} \mathrm{BrP}_{-} \mathrm{SH}_{2}(\mathrm{P} 1)$ & $0.1411(0.1425)$ & $0.2249(0.2288)$ & $(380.2)$ & 0.3280 & 166.2 \\
\hline $\mathrm{H}_{2} \mathrm{FP}-\mathrm{SHF}(\mathrm{P} 2, \mathrm{P} 3)$ & $0.1415(0.1429)$ & $0.1629(0.1679)$ & (760.4) & 0.3096 & 170.0 \\
\hline $\mathrm{H}_{2} \mathrm{ClP}-\mathrm{SHF}(\mathrm{P} 2)$ & $0.1412(0.1426)$ & $0.2086(0.2123)$ & $(487.9)$ & 0.3233 & 167.8 \\
\hline $\mathrm{H}_{2} \mathrm{BrP}-\mathrm{SHF}(\mathrm{P} 2)$ & $0.1412(0.1425)$ & $0.2244(0.2282)$ & $(387.2)$ & 0.3236 & 168.5 \\
\hline $\mathrm{H}_{2} \mathrm{ClP}-\mathrm{SHCl}(\mathrm{P} 3)$ & $0.1412(0.1425)$ & $0.2087(0.2125)$ & $(485.2)$ & 0.3232 & 166.9 \\
\hline $\mathrm{H}_{2} \mathrm{BrP}-\mathrm{SHBr}(\mathrm{P} 3)$ & $0.1412(0.1425)$ & $0.2246(0.2285)$ & (383.6) & 0.3220 & 167.9 \\
\hline Chalcogen bond & $L(\mathrm{SH}) / \mathrm{nm}$ & $L(\mathrm{SY}) / \mathrm{nm}$ & $v(\mathrm{SY}) / \mathrm{cm}^{-1}$ & $L(\mathrm{P} \cdots \mathrm{S}) / \mathrm{nm}$ & $\angle \mathrm{YSP} /\left(^{\circ}\right)$ \\
\hline HFS & $0.1339(0.1353)$ & $0.1634(0.1678)$ & 797.8(781.8) & & \\
\hline HClS & $0.1338(0.1352)$ & $0.2039(0.2077)$ & $550.4(524.7)$ & & \\
\hline $\mathrm{HBrS}$ & $0.1339(0.1353)$ & $0.2188(0.2227)$ & $441.9(417.5)$ & & \\
\hline HFS- $\mathrm{PH}_{3}(\mathrm{~S} 1)$ & $0.1336(0.1350)$ & $0.1670(0.1705)$ & 684.8(701.6) & 0.2737 & 173.9 \\
\hline $\mathrm{HClS} \mathrm{PH}_{3}(\mathrm{~S} 1)$ & $0.1336(0.1350)$ & $0.2062(0.2097)$ & 507.1(487.7) & 0.3079 & 173.1 \\
\hline $\mathrm{HBrS}-\mathrm{PH}_{3}(\mathrm{~S} 1)$ & $0.1336(0.1350)$ & $0.2208(0.2246)$ & (389.6) & 0.3121 & 173.2 \\
\hline HFS-PH ${ }_{2} \mathrm{~F}(\mathrm{~S} 2, \mathrm{~S} 3)$ & $0.1335(0.1351)$ & $0.170(0.1702)$ & (705.3) & 0.2463 & 176.7 \\
\hline HClS- $\mathrm{PH}_{2} \mathrm{~F}(\mathrm{~S} 2)$ & $0.1337(0.1351)$ & $0.2061(0.2091)$ & $(497.0)$ & 0.3026 & 176.7 \\
\hline $\mathrm{HBrS}-\mathrm{PH}_{2} \mathrm{~F}(\mathrm{~S} 2)$ & $0.1337(0.1351)$ & $0.2207(0.2241)$ & (396.9) & 0.3074 & 177.0 \\
\hline HClS-PH ${ }_{2} \mathrm{Cl}(\mathrm{S} 3)$ & $0.1337(0.1351)$ & $0.2059(0.2092)$ & (493.8) & 0.3026 & 177.3 \\
\hline $\mathrm{HBrS}-\mathrm{PH}_{2} \mathrm{Br}(\mathrm{S} 3)$ & $0.1338(0.1352)$ & $0.2205(0.2242)$ & (392.9) & 0.3064 & 177.8 \\
\hline
\end{tabular}

$L$ : bond length: $v$ : frequency. The results in brackets are calculated at MP2/aug-cc-pVDZ level, the other data are calculated at MP2/aug-cc-pVTZ level. 